Persecutory ideation and anomalous perceptual experiences in the context of the COVID-19 outbreak in France: what's left one month later?

\title{
COVID-19 AND PSYCHOTIC EXPERIENCES
}

Catherine Bortolon ${ }^{1,2}$, Delphine Capdevielle ${ }^{3,4}$, Julien Dubreucq ${ }^{2} \&$ Stéphane Raffard $^{3,5}$

${ }^{1}$ Laboratoire Inter-universitaire de Psychologie : Personnalité, Cognition et Changement Social - Université Grenoble Alpes, Grenoble, France.

${ }^{2} \mathrm{C} 3 \mathrm{R}$ - Réhabilitation psychosociale et remédiation cognitive, Centre Hospitalier Alpes Isère, Grenoble, France.

${ }^{3}$ University Department of Adult Psychiatry, La Colombière Hospital, CHU Montpellier, Montpellier, France.

${ }^{4}$ University of Montpellier, Inserm U 1061, Montpellier, France

${ }^{5}$ Univ Paul Valéry Montpellier 3, Univ. Montpellier, EPSYLON EA 4556, 34000 , Montpellier, France

Correspondence concerning this article should be addressed to Dr. Catherine Bortolon, Email: catherine.bortolon@univ-grenoble-alpes.fr, and Pr. Stéphane Raffard, PhD, Email: $\underline{\text { s- }}$ raffard@ chu-montpellier.fr 


\begin{abstract}
Aims: Beyond the effects of the coronavirus pandemic on the public's health, the length of lockdown and its possible psychological impacts on populations around the world is heavily debated. However, the consequences of lockdown on psychotic symptoms have not yet been investigated.
\end{abstract}

Methods: An online survey was run from April 13 to May 11, 2020; a total of 728 French subjects from the general population participated. We assessed the perceived impact of the COVID-19 outbreak, length of self-isolation, diagnosis/symptoms/hospitalisation related to the COVID-19 (oneself and family). Paranoid ideations and anomalous perceptual perceptions were assessed via the Paranoia Scale and the Cardiff Anomalous Perceptions Scale. Measures of negative affect, loneliness, sleep difficulties, jumping to conclusion bias, emotion regulation, and perseverative thinking were also included.

Results: Final regression model for paranoia indicated that socio-demographic variables, loneliness, cognitive bias, anxiety, repetitive thoughts and hallucinations were associated with paranoia $\left(\mathrm{R}^{2}=0.43\right)$. For hallucinations, clinical variables as well as the quality of sleep, behavioural activation, repetitive thoughts, and paranoia were associated with hallucinations in our sample $\left(\mathrm{R}^{2}=0.27\right)$. Neither length of self-isolation nor the perceived impact of the COVID-19 pandemic were associated with psychotic experiences in the final models. Conclusions: No evidence was found between a significant impact of self-isolation on psychotic symptoms in the general population in France one month after the lockdown. It nevertheless confirms the preeminent role of several factors previously described in the maintenance and development of psychotic symptoms in the context of a pandemic and lockdown measures.

Key-words: COVID-19; Lockdown; Self-isolation; Hallucinations; Paranoia 


\section{Introduction}

The pandemic of the novel coronavirus disease (COVID-19) has now affected millions of people, including people in France. French people have been under lockdown from Mar 15 until May 11 leading, in many cases, to abrupt changes in 'people's lives, such as working from home or under stressful circumstances, loss of employment, and social and physical distancing with outstanding consequences for 'people's mental and physical health (Cava et al 2005, Marjanovic et al 2007). In addition to the uncertainty and stress of the global outbreak, questions about the effects of quarantining on mental and psychological health have quickly emerged.

A recent review by Brooks and colleagues (2020) published at Lancet summarise the currently available knowledge on the psychological effects of quarantine. Mental health issues mostly explored were post-traumatic disorder symptoms, depression, anxiety, loneliness, insomnia and other negative emotions in general (e.g., anger, frustration and boredom) (Brooks et al 2020). Nevertheless, all studies reviewed by the authors were carried out in situations of quarantine where the person was isolated due to the infected with SARS, Ebola, H1N1 influenza pandemic, among others.

A few studies have been carried out in these last few weeks to understand the specific impact of the COVID-19 quarantine on mental health. For instance, Pancani and colleagues (2020) showed, in a sample of 1006 Italians currently locked down, that the length of isolation is associated with worse mental health including higher levels of depression, unworthiness, and helplessness. Another study by Zhang et al. (2020) showed that individuals who had to stop working during the outbreak are more vulnerable to mental and physical health issues. As for another example, Shevlin and colleagues (2020) who found in a sample of 2025 UK adults increased levels of anxiety, depression and trauma compared to previous populations studies, but not a dramatic increase. Factors that predicted higher levels of mental health symptoms 
included young age, high estimates of personal risk, low income, loss of revenue, as well as pre-existing health conditions.

Surprisingly, no study has yet explored whether the current situation or its perceived impact can exacerbate psychotic experiences such as paranoid ideas and hallucinations and/or contribute to the emergence of these experiences in the general population. This fact is even more surprising if one considers that factors that have been the focus of attention of the studies described above such as sleep-related problems (Reeve et al 2015), aggravated social isolation and loneliness as a result of the lockdown (Lamster et al 2017, Michalska da Rocha et al 2018), emotional distress (Delespaul et al 2002, Lincoln et al 2009) and worry/rumination (Badcock et al 2011, Startup et al 2016) are significant predictors of psychotic symptoms in clinical, but also in non-clinical populations (Butler et al 2019, Reeve et al 2018). For instance, many studies have shown that isolation are associated with increased frequency of hallucinatory experiences (Davis et al 1961, Kellerman et al 1977, Zubek et al 1961). Likewise, Grassian (1983) found that inmates exposed to solitary confinement described perceptual distortions and hallucinations. Moreover, sleep deprivation has been found to increase paranoia and hallucinations in a sample of non-clinical volunteers (Reeve et al 2018).

Indeed, the vulnerability-stress model emphasises the role of stress in the emergence of psychotic experiences (Lecomte et al 2019, Nuechterlein et al 1994). Many studies have found consistent evidence showing that emotional reactivity to stress is a vulnerability marker for psychosis (Myin-Germeys and van Os 2007). Moreover, theoretical models of hallucinations highlight the fundamental role of negative emotions in the emergence and maintenance of auditory hallucinations (Birchwood 2003, Freeman and Garety 2003, Morrison 2001). Similarly, theoretical models of paranoia place anxiety and worry as core predictors of paranoia (Startup et al 2016). Therefore, the recent outbreak of COVID-19, the lockdown and the 
exposure to sensationalised news about crises are here important stress-inducing events that can predispose individuals to paranoia and hallucinations.

The goal of the present study is, therefore, to add to the current literature information about the impact of the COVID-19 pandemic and lockdown on psychotic experiences. More specifically, we sought to study which factors are associated with paranoid ideas and hallucinations during the lockdown period. Our main correlates of psychotic experiences can be divided into five groups. We adopted Freeman and Garety's model (2014) of paranoia and their general model of psychotic symptoms (Garety et al 2001) to guide the inclusion of our variables. According to their models, when facing stressful situations (e.g., COVID-19, lack of sleep, social isolation, etc.), individuals might interpret internal and external events in a different way because of different cognitive (i.e., jumping to conclusion) and emotional (i.e., anxiety, repetitive thoughts, emotion regulation strategies) factors, which ultimately will result in increased paranoid ideas. Likewise, stressful situations are suggested to trigger hallucinations, notably through cognitive and affective changes. Cognitive and emotional processes associated with hallucinations include repetitive thinking and intrusive thoughts (Badcock et al 2011, Gracie et al 2007), negative affect such as depression and anxiety (Delespaul et al 2002, Hartley et al 2014), and experiential avoidance (Goldstone et al 2011, Langer et al 2010).

Therefore, we first included the social-demographic variables (e.g., age, gender and level of education) as well as clinical variables (e.g., cannabis consumption, previous mental health disorders diagnosis). Second, we assessed the length and perceived impact of the lockdown and participant exposure to the virus (COVID-19 related triggers). Third, we also added measures of sleep quality, level of activity and loneliness during the quarantine (Contextual triggers). Forth, we included a measure of jumping to conclusion bias (JTC), which describes individual's tendency to make a hasty decision on the basis of little evidence. JTC is the reasoning bias most consistently associated with delusions and particularly paranoid 
ideations in both clinical (Dudley et al 2016) and non-clinical samples (Freeman et al 2008). Fifth, in light of previous studies highlighting the role of emotional processes we included measures of emotion regulation (i.e., experiential avoidance, cognitive restructuring and catastrophisation), emotional distress (i.e., anxiety) and repetitive thoughts. Finally, we considered the role of paranoia (for the hallucination model) and hallucinations (for the paranoia model) since they often appear together.

First, we hypothesise that emotion regulation (emotional avoidance), negative affect, level of activity, loneliness, sleep quality, perception of the impact of the COVID-19 pandemic, and length of self-isolation (number of days since the beginning of lockdown in France) will be associated with hallucinations. Second, we hypothesise that emotion regulation (catastrophising and reappraisal), cognitive bias, negative affect, worry, level of activity, loneliness, sleep quality, length of self-isolation, and perception of the impact of the COVID-19 pandemic will be associated with paranoia.

The hypothesis of the study was pre-registered (https://osf.io/gxy79/). Based on the literature, we made two corrections in our second hypothesis: we added both sleep quality (Reeve et al 2015) and loneliness (Lamster et al 2017) as predictor of paranoia.

\section{Method}

\section{Participants}

Our sample was recruited in the general population through online social media, through university websites and via acquaintances of the authors with a snowball procedure. Moreover, each participant was requested to invite at least three friends, family members, or acquaintances to take part in the study. Inclusion criteria consisted of being 18 years old or more and being fluent in French. 
In total, 1154 participants started the study, but only 733 complete all scales, including the Paranoia Scale. Fewer participants completed both the measure of paranoia and hallucinationlike experiences $(\mathrm{N}=719)$. Participants who failed at least two validation questions (6 questions were included in the study) were excluded $(\mathrm{N}=5)$. In total, 728 participants who consented to participate and complete at least the Paranoia Scale were included in the final sample.

Ethical clearance was provided by Centre Hospitalier Universitaire de Montpellier, IRBMTP_2020_04_202000449.

\section{Measures}

Socio-demographic variables: Participants completed a series of questions about the socio-demographic characteristics, including sex, age, nationality, education level, professional status, and family situation.

Clinical measures: Participants provided information about current and past mental health diagnosis, mental health treatment and drugs consumption.

COVID-19 related measures: Participants were requested to complete different questions about their perception of the lockdown and security measures as well as the impact of the lockdown. The latter question covered different domains, including the effect of the lockdown on French's lifestyle, economy, mental health and health system, as well as its impact on one's psychological and physical health and finances. Participants were requested to rate whether they agree with the statement, and if they did, they also evaluate their levels of preoccupation and distress. Finally, participants were requested to assess whether they or someone close to them was infected by the COVID-19 and/or hospitalised.

The Behavioural Activation for Depression Scale - BADS (Kanter et al 2007) is a 10item scale assessing behavioural activation. Each item is rated on a 7-point scale (from 0 to 6 ). For the total scale, higher scores indicate higher behavioural activation. 
The UCLA Loneliness Scale (Russell 1996) is a 20-item measure that assesses how often a person feels disconnected from others. Items are rated on a 4-point rating scale ranging from 1 (never) to 4 (always). Higher scores indicate higher levels of loneliness.

The Pittsburgh Sleep Quality Index - PSQI (Buysse et al 1989) is a subjective measure of sleep. It measures seven domains: subjective sleep quality, sleep latency, sleep duration, habitual sleep efficiency, sleep disturbances, use of sleep medication, and daytime dysfunction over the last month. Scoring of the answers is based on a 0 to 3 Likert Scale. In the current study, we assessed only two domains (three questions): use of sleep medication and daytime dysfunction.

The Brief Experiential Avoidance Questionnaire - BEAQ (Gámez et al 2014) is a selfreport questionnaire designed to measure experiential avoidance, that is, the unwillingness to remain in contact with distressing emotions, thoughts and other experiences (Hayes, Strosahal, $\&$ Wilson, 2012). Items are rated on a 6-point scale ranging from 1 (strongly disagree) to 6 (strongly agree). This brief version is composed of 15 items.

The Cognitive Emotional Regulation Questionnaire - CERQ (Garnefski and Kraaij 2007) is a 36-item scale designed to evaluate nine cognitive strategies used to regulate emotions in response to negative or unpleasant events. In the present study, we focused on two: Catastrophizing and Putting into perspective (or cognitive reappraisal) because previous studies have found that catastrophising and impaired cognitive reappraisal are important correlated of paranoia (Startup et al 2007, Westermann et al 2014).

The Perseverative Thinking Questionnaire - PTQ (Ehring et al 2011). The PTQ was developed to propose a transdiagnostic measure of the core characteristics of negative repetitive thinking (RNT), such as the repetitiveness, the intrusiveness, or the difficulty of disengaging, and the perceived unproductiveness of RNT. The PTQ is a 15-item scale. Items are rated on a 5-point scale ranging from 0 (never) to 4 (almost always). 
The Cognitive bias questionnaire for Psychosis - CBQp (Peters et al 2013). The CBQp was developed to capture 5 cognitive distortions including jumping to conclusions,For the purpose of the study, we focused on the six items of the CBQp related to the jumping to conclusion bias (JTC). The potential range of scores was 6-18.

The Depression Anxiety and Stress Scale - DASS (Lovibond \& Lovibond, 1994) is a scale measuring negative affect including depression, anxiety and stress. Each item is scored on a 4-point scale (0-never; 4-all the time). Higher scores indicate greater levels of negative affect.

The Paranoia Scale - PS (Fenigstein and Vanable 1992) is a 20-item self-report questionnaire developed to assess the frequency of subclinical levels of paranoia. It is scored on a 1-5 Likert scale with scores ranging from 20-100.

The Cardiff Anomalous Perceptions Scale-CAPS (Bell et al 2006) is a self-report scale designed to assesses a range of hallucination-like experiences. It consists of 32 items, with yes/no as response options.

The Validation questions. Five items were added to detect random completion or attention lapses (e.g., "please answer 4 to this question"), lies (issued from the Eysenck Personality Questionnaire-Revised; (Eysenck et al 1984) and simulation of psychotic symptoms (Moritz et al 2013).

For all measures, participants were requested to consider only the period of lockdown while completing the questionnaires.

All measures employed in this study are available here: https://osf.io/gxy79/. This study was pre-registered.

\section{Statistical Analyses}


Mean, and the standard deviation was calculated for all variables (see Table 1).

Subsequently, correlations and multiple linear regression analysis were used to test the relationship between the dependent variables (Paranoia and Hallucinations) and the independent variables. Regressions were performed in R with the $\operatorname{lm}$ (linear model) function. Moreover, we perform, regression diagnostics including tests of normality of residuals (Normal Probability Plot), homoscedasticity (Plot of residuals versus predicted value), independence (Durbin-Watson statistic) of Residuals, Non-linearity, and multicollinearity (VIF < 2). The function Normalize() was employed to improve the model. Dummy variables were created for the categorical variables. The significance level for our analyses was set at $p$ $<0.05$. All statistical analyses were performed using the R Studio.

\section{Results}

\section{Descriptive Variables}

Descriptive characteristics of the sample are presented in Table 1 (for continuous variables) and Table 2 (for categorical variables).

Insert Table 1 and 2 here

\section{Correlates of Paranoia}

Correlation between paranoia and the other variables are presented in Table 3. After Benjamini and Hochberg correction, paranoia was found to be statistically correlated to age, education, perceived impact of lockdown, sleep quality, loneliness, experiential avoidance, behavioural activation, catastrophisation, repetitive thoughts, cognitive bias, negative affect and hallucinations. 
For the categorical variables entered in the model, refer to Table 4. Mann-whitey test showed statistically significant differences in paranoia when considering the following independent variables: Diagnosis of Mental disorder, Past Mental disorder, Currently seeing a mental health professional, and Currently taking medication for a mental disorder.

Insert here Table 3 and 4

\section{Regression}

Seven different models were tested for paranoia. First, we entered only the socio-demographic variables (Model 1). Although sex was not related to paranoia, we decided to keep it in the final model. Nevertheless, we decided to keep sex in the regression model due to the important number of female (vs male) in our sample. Second, we also included clinical variables (Model 2).

Third, we entered the COVID-19 related triggers (Model 3). Forth, in Model 4, quality of sleep during the quarantine, behavioural activation and loneliness were added (Contextual triggers). Fifth, the cognitive factors were included (Model 5) followed by the emotional ones (Model 6). Finally, in Model 7, we also included paranoia. The choice of anxiety was based on theoretical grounds (Freeman \& Garety, 2014) and in order to avoid collinearity issues. Entering the total score of the DASS resulted in multicollinearity.

As it can be seen in Table 5, most clinical and COVID-19 related variables were no longer statistically associated with paranoia once we entered in the model the affective and cognitive processes that are commonly associated with paranoia. Together with the socio-demographic variables, loneliness, cognitive bias, anxiety, repetitive thoughts and hallucinations were associated with paranoia. 
Insert here Table 5

\section{Correlates of Hallucinations and Other unusual experiences}

See Table 3 for the correlation analyses for Hallucinations and other unusual perceptual experiences. After Benjamini and Hochberg correction, hallucinations were found to be statistically correlated to all variables except for the length of self-isolation and reappraisal.

For the categorical variables entered in the model, refer to Table 4. Mann-whitey test showed statistically significant differences in hallucinations when considering the following independent variables: Diagnosis of Mental disorder, Past Mental disorder, Currently seeing a mental health professional, and Currently taking medication for a mental disorder.

Insert here Table 6

\section{Regression}

The same steps adopted to test the models for paranoia were adopted here (Table 5). The only difference here is that paranoia was entered in the final step. Again, we choose to include here only anxiety considering previous studies showing that it is "the most prominent emotion during hallucinations" and that "reports of anxiety intensity exceeded baseline levels before the first report of auditory hallucinations" (Delespaul, et al., 2002, abstract). Moreover, we decided to keep only one of the DASS scores to avoid multicollinearity problems. 
As it can be seen in Table 6, socio-demographic and COVID-19 related variables were not statistically associated with hallucinations once we entered in the model the factors that are commonly associated with hallucinations were considered (see Model 4 and 6). In Model 7, diagnosis of a mental disorder, currently taking medication, quality of sleep, behavioural activation, repetitive thoughts, and paranoia were associated with hallucinations in our sample. Having a diagnosis was associated with having more hallucinatory experiences. Medication use was negatively associated with hallucinations indicating that those not consuming medication also reported less hallucinatory experiences. Lower sleep quality (higher score) was associated with reporting more hallucinations. Surprisingly, behavioural activation was positively associated with hallucinations indicating that those individuals who were engaging in more activities in their daily life also reported more hallucinations. Repetitive intrusive thoughts, anxiety and paranoia were positively associated with hallucinations.

Insert here table 6

Influential outliers were found in the model as well as issues with the normality of residuals. Exclusion of outliers did not improve the model; neither changed the results. We decided to report the model without excluding them. Nevertheless, caution is necessary when generalising our findings.

\section{Discussion}

The actual pandemic of the new Coronavirus disease (COVID-19) has led many countries to impose lockdown to a large proportion of their population to avoid spreading the 
disease further. France, for example, has been put on lockdown for eight weeks between $17 / 03 / 2020$ and 11/05/2020. As indicated by a recent review (Brooks et al 2020), past experiences revealed that more extended quarantine is associated with poorer psychological outcomes and generally marked by increased incidence for various psychiatric symptoms, particularly post-traumatic stress symptoms, depression, or sleep disturbances. Also, and as discussed elsewhere, pandemic conditions are «unique circumstances to determine causal mechanisms associated with poor mental health » outside the laboratory (Holmes et al 2020). Surprisingly, no study has yet explored whether the length of lockdown and its perceived impact can exacerbate psychotic experiences, including paranoid ideas and hallucinations in the general population. This is despite the report of several cases of brief psychotic disorder triggered or exacerbated by the fear of the coronavirus (Fischer et al 2020).

Our study had two main objectives. First, to study the impact of the length of the quarantine and perceived impact associated with the COVID-19 pandemic on paranoid ideation and hallucinations in the general population. Second, to examine an integrative model of psychotic symptoms in the context of the lockdown imposed by COVID-19 pandemic.

Overall, despite cumulative evidence and scientific reports that the COVID-19 pandemic and, particularly, self-isolation affects mental health and particularly financial worry, y or loneliness in the general population (Asmundson and Taylor 2020, Tull et al 2020), our results did not support such a conclusion regarding paranoid ideations and hallucinations and other unusual perceptual experiences.

Firstly, the average score of paranoid ideations $(\mathrm{M}=35.58 ; \mathrm{SD}=12.32)$ and hallucinations $(\mathrm{M}=3.73$; $\mathrm{SD}=4.68)$ was similar or inferior to those previously found in the general community populations outside pandemic conditions. For instance, for paranoia, Hajdúk et al. (2019), in UK, reported a mean score of 39.35 (SD = 12.39), while in another study by Pinto-Gouveia and colleagues (2014) conducted in Portugal, they found the mean 
score for the Paranoia Scale of 44.20 (SD = 10.61). Regarding hallucinations and other unusual perceptual experiences, the validation study of the CAPS (Bell et al 2006), in UK, found a mean of $7.3(\mathrm{SD}=5.8$; participants were much younger in their study, mean age $=21.6, \mathrm{SD}=5.4)$. As for another example, Moseley and colleagues (in preparation), in a large multi-country sample (including France), reported a mean for the CAPS of 4.68. Thus, there is no evidence of abnormal levels of psychotic symptoms in our sample one month after the confinement.

Secondly, the length of self-isolation was found to be correlated with neither paranoia ideas nor hallucinations. Moreover, contrary to our hypothesis, even though the perceived impact of the COVID-19 pandemic was initially associated with psychotic symptoms, in our regression analyses, their effect was small and became non-significant once we considered other contextual factors that are commonly associated with hallucinations and paranoia such as sleep difficulties (Reeve et al 2015), and loneliness (Lamster et al 2017). Moreover, neither the presence of COVID-19 symptoms nor the presence of a family member with a diagnosis was associated with reporting more psychotic-like experiences. Unfortunately, due to the small number of participants reporting a diagnosis of COVID-19 or hospitalisations due to it, we could not test whether it could be associated with increased psychotic symptoms.

Regarding our paranoia model, the main correlates that explain $44 \%$ of the variance of paranoid ideations were loneliness, jumping to conclusions bias (failure to consider alternative explanations, anxiety, experiential avoidance and hallucinations. These findings partially support our hypothesis. Age, sex and education were also correlated to paranoia (Freeman et al 2008, Freeman and Garety 2014). Our model of paranoia during the COVID-19 pandemic fits well with the threat anticipation model (Freeman 2007, Freeman and Garety 2014), which proposes a multi-factorial account of the formation and maintenance of persecutory delusions comprising affective processes and especially anxiety, worry, and interpersonal sensitivity (Freeman and Garety 2003); anomalous experiences, such as hallucinations and perceptual 
anomalies; reasoning biases, particularly jumping to conclusions and belief inflexibility; and finally social factors, such as adverse or stressful events and environments. One of the main contributions of our findings is that we reproduce and extend the threat anticipation model under exceptional conditions corresponding to the defining global health crisis of our time.

Concerning our model for hallucinations, the main correlates were anxiety, a current diagnosis of mental disorder, and currently taking medication. The other correlates were reduced sleep quality, higher levels of behavioural activation, repetitive negative thinking and paranoia ideation that all together explain $27 \%$ of hallucination. These results corroborate some of our hypothesis and also previous studies showing the importance of anxiety (Delespaul et al 2002), quality of sleep (Reeve et al 2015), and repetitive thinking (Badcock et al 2011, Gracie et al 2007) as important factors contributing to hallucinatory experiences. Surprisingly, behavioural activation was positively associated with hallucinations in the regression model (although not in the correlation analyses). Multicollinearity problems were not detected. Theoretically, these results contradict the idea that individuals who are more engaged in meaningful activities might report fewer hallucinations in daily life. The interaction with another variable might be contributing to this finding, despite the absence of multicollinearity problems.

Conclusions. No evidence was found between a significant association between length of selfisolation and psychotic symptoms in the general population in France. Our findings indicate that although the perceived impact of lockdown is associated with increased paranoid ideas and hallucinatory experiences, other factors are more important contributors to our final model. Contrary to what one might have feared (Troyer et al 2020), the COVID-19 pandemic does not appear to be a direct risk factor for psychotic symptomology, at least in the French population with an average lockdown length above one month. One could, however, hypothesise that the 
pandemic and the lockdown might maximise other relevant risk factors. Indeed, our study confirms the preeminent role of several cognitive, emotional and contextual factors previously described in the maintenance and development of psychotic symptoms in the context of an epidemic and lockdown measures.

Limits. Our study has several limits. First, this is a cross-sectional study, and it is still too soon to forecast the medium-term consequences of the correlates. Second, our sample comes from the general population without restricted access to the internet. Our results need to be confirmed in populations that are socially or economically fragile, belonging to an immigrant or minority group of the population with a higher risk of developing paranoid ideations (Lopes, Bortolon, \& Jaspal, under review). Similarly, even if it was not the aim of our study, our results cannot be generalised to clinical populations. Future studies are needed to explore specifically the short and long-term consequences of the correlates on psychotic symptoms in populations facing difficulties in accessing timely health services (Yao et al 2020).

\section{Acknowledgements}

We would like to thank every single participant of this study for taking the time to complete our measures during this difficult period.

\section{Financial Support}

This research received no specific grant from any funding agency, commercial or not-forprofit sectors.

\section{Conflicts of Interest: None}

\section{Ethical Standards}

The authors assert that all procedures contributing to this work comply with the ethical standards of the relevant national and institutional committees on human experimentation and with the Helsinki Declaration of 1975, as revised in 200. Ethical clearance was provided by Centre Hospitalier Universitaire de Montpellier, IRB-MTP_2020_04_202000449. 


\section{References}

Asmundson GJ, Taylor S (2020) How health anxiety influences responses to viral outbreaks like COVID-19: What all decision-makers, health authorities, and health care professionals need to know. Journal of Anxiety Disorders 71, 102211-102211.

Badcock JC, Paulik G, Maybery MT (2011) The role of emotion regulation in auditory hallucinations. Psychiatry research 185(3), 303-308.

Bell V, Halligan PW, Ellis HD (2006) The Cardiff Anomalous Perceptions Scale (CAPS), a new validated measure of anomalous perceptual experience. Schizophrenia bulletin. Oxford University Press 32(2), 366-377.

Birchwood M (2003) Pathways to emotional dysfunction in first-episode psychosis. The British Journal of Psychiatry. Cambridge University Press 182(5), 373-375.

\section{Brooks SK, Webster RK, Smith LE, Woodland L, Wessely S, Greenberg N, Rubin GJ (2020) The psychological impact of quarantine and how to reduce it: rapid review of the evidence. The Lancet. Elsevier.}

Butler R, Berry K, Ellett L, Bucci S (2019) An experimental investigation of the impact of critical and warm comments on state paranoia in a non-clinical sample. Journal of behavior therapy and experimental psychiatry 62, 30-37.

Buysse DJ, Reynolds CF, Monk TH, Berman SR, Kupfer DJ (1989) The Pittsburgh Sleep Quality Index: a new instrument for psychiatric practice and research. Psychiatry res 28(2), $193-213$. 
Cava MA, Fay KE, Beanlands HJ, McCay EA, Wignall R (2005) The experience of quarantine for individuals affected by SARS in Toronto. Public Health Nursing. Wiley Online Library 22(5), 398-406.

Davis JM, McCourt WF, Courtney J, Solomon P (1961) Sensory deprivation: The role of social isolation. Archives of general psychiatry. American Medical Association 5(1), 84-90.

Delespaul P, Devries M, van Os J (2002) Determinants of occurrence and recovery from hallucinations in daily life. Social psychiatry and psychiatric epidemiology 37(3), 97-104.

Dudley R, Taylor P, Wickham S, Hutton P (2016) Psychosis, delusions and the "jumping to conclusions" reasoning bias: a systematic review and meta-analysis. Schizophrenia bulletin. Oxford University Press US 42(3), 652-665.

Ehring T, Zetsche U, Weidacker K, Wahl K, Schönfeld S, Ehlers A (2011) The Perseverative Thinking Questionnaire (PTQ), Validation of a content-independent measure of repetitive negative thinking. Journal of behavior therapy and experimental psychiatry. Elsevier 42(2), 225-232.

Eysenck SB, Barrett P, Eysenck HJ (1984) Eysenck personality questionnaire-revised. Pergamon Press.

Fenigstein A, Vanable PA (1992) Paranoia and self-consciousness. Journal of personality and social psychology. American Psychological Association 62(1), 129.

Fischer M, Coogan A, Faltraco F, Thome J (2020) COVID-19 paranoia in a patient suffering from schizophrenic psychosis-a case report. Psychiatry Research. Elsevier 288, 113001. 
Freeman D (2007) Suspicious minds: the psychology of persecutory delusions. Clinical psychology review 27(4), 425-457.

Freeman D, Garety P (2014) Advances in understanding and treating persecutory delusions: a review. Social psychiatry and psychiatric epidemiology. Springer 49(8), 1179-1189.

Freeman D, Garety PA (2003) Connecting neurosis and psychosis: the direct influence of emotion on delusions and hallucinations. Behaviour research and therapy 41(8), 923-947.

Freeman D, Pugh K, Garety P (2008) Jumping to conclusions and paranoid ideation in the general population. Schizophrenia research. Elsevier 102(1-3), 254-260.

Gámez W, Chmielewski M, Kotov R, Ruggero C, Suzuki N and Watson D (2014) The brief experiential avoidance questionnaire: development and initial validation. Psychological assessment. American Psychological Association 26(1), 35.

Garety PA, Kuipers E, Fowler D, Freeman D, Bebbington P (2001) A cognitive model of the positive symptoms of psychosis. Psychological medicine 31(2), 189-195.

Garnefski N, Kraaij V (2007) The cognitive emotion regulation questionnaire. European Journal of Psychological Assessment. Hogrefe \& Huber Publishers 23(3), 141-149.

Goldstone E, Farhall J, Ong B (2011) Life hassles, experiential avoidance and distressing delusional experiences. Behaviour Research and Therapy 49(4), 260-266.

\section{Gracie A, Freeman D, Green S, Garety P, Kuipers E, Hardy A, Ray K, Dunn G,}

Bebbington P, Fowler D (2007) The association between traumatic experience, paranoia and hallucinations: a test of the predictions of psychological models. Acta Psychiatrica Scandinavica 116(4), 280-289. 
Hajdúk M, Klein HS, Harvey PD, Penn DL, Pinkham AE (2019) Paranoia and interpersonal functioning across the continuum from healthy to pathological-Network analysis. British Journal of Clinical Psychology. Wiley Online Library 58(1), 19-34.

Hartley S, Haddock G, e Sa DV, Emsley R, Barrowclough C (2014) An experience sampling study of worry and rumination in psychosis. Psychological Medicine 44(8), 16051614.

Holmes EA, O’Connor RC, Perry VH, Tracey I, Wessely S, Arseneault L, Ballard C, Christensen H, Silver RC, Everall I, Ford T (2020) Multidisciplinary research priorities for the COVID-19 pandemic: a call for action for mental health science. The Lancet Psychiatry. Elsevier.

Jaya ES, Hillmann TE, Reininger KM, Gollwitzer A, Lincoln TM (2017) Loneliness and psychotic symptoms: The mediating role of depression. Cognitive therapy and research. Springer 41(1), 106-116.

Kanter JW, Mulick PS, Busch AM, Berlin KS, Martell CR (2007) The Behavioral Activation for Depression Scale (BADS), psychometric properties and factor structure. Journal of Psychopathology and Behavioral Assessment. Springer 29(3), 191.

Kellerman J, Rigler D, Siegel SE (1977) The psychological effects of isolation in protected environments. The American journal of psychiatry. American Psychiatric Assn.

Lamster F, Nittel C, Rief W, Mehl S, Lincoln T (2017) The impact of loneliness on paranoia: An experimental approach. Journal of behavior therapy and experimental psychiatry. Elsevier 54, 51-57. 


\section{Langer ÁI, Cangas AJ, Pérez-Moreno PJ, Carmona JA, Gallego J (2010) Relation}

between experiential avoidance, hallucination-like experiences and clinical symptoms, in a non-clinical spanish sample. Journal of Cognitive \& Behavioral Psychotherapies 10(2).

\section{Lecomte T, Potvin S, Samson C, Francoeur A, Hache-Labelle C, Gagné S, Boucher J,} Bouchard M, Mueser KT (2019) Predicting, preventing symptom onset and relapse in schizophrenia-A metareview of current empirical evidence. Journal of abnormal psychology. American Psychological Association 128(8), 840.

Lincoln T, Peter N, Schäfer M, Moritz S (2009) Impact of stress on paranoia: an experimental investigation of moderators and mediators. Psychological medicine. Cambridge University Press 39(7), 1129-1139.

Marjanovic Z, Greenglass ER, Coffey S (2007) The relevance of psychosocial variables and working conditions in predicting nurses' coping strategies during the SARS crisis: an online questionnaire survey. International journal of nursing studies. Elsevier 44(6), 991-998.

Michalska da Rocha B, Rhodes S, Vasilopoulou E, Hutton P (2018) Loneliness in psychosis: a meta-analytical review. Schizophrenia bulletin. Oxford University Press US $44(1), 114-125$.

Moritz S, Van Quaquebeke N, Lincoln TM, Köther U, Andreou C (2013) Can we trust the internet to measure psychotic symptoms? Schizophrenia research and treatment 2013.

Morrison AP (2001) The interpretation of intrusions in psychosis: an integrative cognitive approach to hallucinations and delusions. Behavioural and Cognitive Psychotherapy 29(3), $257-276$. 
Myin-Germeys I, van Os J (2007) Stress-reactivity in psychosis: evidence for an affective pathway to psychosis. Clinical psychology review. Elsevier 27(4), 409-424.

\section{Nuechterlein KH, Dawson ME, Ventura J, Gitlin M, Subotnik KL, Snyder KS, Mintz J} and Bartzokis G (1994) The vulnerability/stress model of schizophrenic relapse: a longitudinal study. Acta Psychiatrica Scandinavica. Wiley Online Library 89, 58-64.

Pancani L, Marinucci M, Aureli N, Riva P (2020) Forced social isolation and mental health: A study on 1006 Italians under COVID-19 quarantine. PsyArXiv.

\section{Peters ER, Moritz S, Schwannauer M, Wiseman Z, Greenwood KE, Scott J, Beck AT,} Donaldson C, Hagen R, Ross K, Veckenstedt R (2013) Cognitive biases questionnaire for psychosis. Schizophrenia bulletin 40(2), 300-313.

Pinto-Gouveia J, Matos M, Castilho P, Xavier A (2014) Differences between depression and paranoia: The role of emotional memories, shame and subordination. Clinical psychology \& psychotherapy. Wiley Online Library 21(1), 49-61.

Reeve S, Emsley R, Sheaves B, Freeman D (2018) Disrupting sleep: the effects of sleep loss on psychotic experiences tested in an experimental study with mediation analysis. Schizophrenia bulletin. Oxford University Press US 44(3), 662-671.

Reeve S, Sheaves B, Freeman D (2015) The role of sleep dysfunction in the occurrence of delusions and hallucinations: a systematic review. Clinical Psychology Review. Elsevier 42, 96-115.

Russell DW (1996) UCLA Loneliness Scale (Version 3), Reliability, validity, and factor structure. Journal of personality assessment. Taylor \& Francis 66(1), 20-40. 


\section{Shevlin M, McBride O, Murphy J, Miller JG, Hartman TK, Levita L, Mason L,}

Martinez AP, McKay R, Stocks TV, Bennett KM (2020) Anxiety, Depression, Traumatic

Stress, and COVID-19 Related Anxiety in the UK General Population During the COVID-19 Pandemic. PsyArXiv.

Startup H, Freeman D, Garety PA (2007) Persecutory delusions and catastrophic worry in psychosis: developing the understanding of delusion distress and persistence. Behaviour research and therapy. Elsevier 45(3), 523-537.

\section{Startup H, Pugh K, Dunn G, Cordwell J, Mander H, Černis E, Wingham G, Shirvell K,}

Kingdon D, Freeman D (2016) Worry processes in patients with persecutory delusions.

British Journal of Clinical Psychology 55(4), 387-400.

Troyer EA, Kohn JN, Hong S (2020) Are we facing a crashing wave of neuropsychiatric sequelae of COVID-19? Neuropsychiatric symptoms and potential immunologic mechanisms. Brain, behavior, and immunity. Elsevier.

Tull MT, Edmonds KA, Scamaldo K, Richmond JR, Rose JP, Gratz KL (2020)

Psychological Outcomes Associated with Stay-at-Home Orders and the Perceived Impact of COVID-19 on Daily Life. Psychiatry Research. Elsevier 113098.

Westermann S, Rief W, Lincoln TM (2014) Emotion regulation in delusion-proneness: Deficits in cognitive reappraisal, but not in expressive suppression. Psychology and Psychotherapy: Theory, Research and Practice. Wiley Online Library 87(1), 1-14.

Yao H, Chen J-H, Xu Y-F (2020) Patients with mental health disorders in the COVID-19 epidemic. The Lancet Psychiatry. Elsevier 7(4), e21. 
Zubek JP, Pushkar D, Sansom W, Gowing J (1961) Perceptual changes after prolonged sensory isolation (darkness and silence). Canadian Journal of Psychology/Revue canadienne de psychologie. University of Toronto Press 15(2), 83. 


\section{Table 1.}

Minimum, Maximum, Mean and Standard Deviation for the categorical variables $(\mathrm{N}=728)$

\begin{tabular}{lcc}
\hline & Mean & SD \\
\hline Age & 44.90 & 15.95
\end{tabular}

Covid-19 related triggers

Perceive impact of the quarantine

$23.36 \quad 5.36$

Associated Preoccupation

25.21

9.97

Associated Stress

$20.05 \quad 11.14$

Length of Isolation

$38.23 \quad 8.42$

\section{Contextual triggers}

Sleep quality

1.99

1.77

Behavioral Activation

31.39

9.42

Loneliness

41.71

8.87

\section{Cognitive and Emotional factors}

Experiential Avoidance

$48.83 \quad 12.65$

Reappraisal

$15.49 \quad 3.62$

Catastrophisation

5.84

2.41

Repetitive thoughts

22.97

13.01

Jumping to conclusion bias

$9.51 \quad 1.74$

Negative Affect

$13.96 \quad 11.33$

Anxiety

3.11

Depression

$4.91 \quad 4.67$




\begin{tabular}{lcc} 
Stress & 5.94 & 4.63 \\
Paranoia & 35.58 & 12.32 \\
Hallucination * & 3.73 & 4.68 \\
\hline
\end{tabular}

Note $: * N=714$

Table 2.

Number $(\mathrm{N})$ and percentage $(\%)$ for the categorical variables

\begin{tabular}{lcc}
\hline & $\mathrm{N}$ & $\%$ \\
\hline Sex & 554 & 76.10 \\
Female & 173 & 23.76 \\
Male & 1 & 0.1 \\
Other & & \\
Education & & \\
Elementary School & 2 & 0.20 \\
Secondary School & 9 & 1.24 \\
High School & 129 & 17.72 \\
Bachelor's Degree & 228 & 31.32 \\
'Master's degree & 295 & 40.52
\end{tabular}




\section{Family Situation}

Single

Married

$222 \quad 30.49$

In a couple

186

25.55

Divorced

Widower

\section{Profession}

Student

Employee

Independent worker

Retired

Unemployed

Incapacity to work

Autre

54

7.42

Nationality

French

Other

\section{Country}

France

Other

14

1.92

Diagnosis of Serious Mental Disorder 
Depression

Anxiety Disorders

Schizophrenia/Psychosis

Bipolar Disorder

Eating Disorder

Personality Disorder

8

Autism Spectrum Disorder

6

7.5

Other

4 5

Prefer not to say

14

1.92

\section{Past Serious Mental Disorder}

No

Yes

Depression

Anxiety Disorders

Schizophrenia/Psychosis

Bipolar Disorder

Eating Disorder

Personality Disorder

Autism Spectrum Disorder

Other
1

0.60

8

4.84 
Currently seeing a mental health professional

No

Yes

$128 \quad 17.58$

Prefer not to say

5

0.69

Currently taking medication

No

Yes

$102 \quad 14.01$

Prefer not to say

7

0.96

Cannabis consumption

No

$698 \quad 95.88$

Yes

30

4.12

Diagnosis of COVID-19

No

Yes

Familiy diagnosed COVID-19

No

Yes

$164 \quad 22.53$

COVID-19 symptoms

No

Yes 
No

Yes

Hospitalised due to COVID-19

No

Yes
712

16 2.20

727

1

0.14 
Table 3.

Spearman correlations for Paranoia and Hallucinations.

\begin{tabular}{|c|c|c|c|c|}
\hline & \multicolumn{2}{|c|}{ Paranoia } & \multicolumn{2}{|c|}{ Hallucination } \\
\hline & $\mathbf{r}$ & $p$-value & $\mathbf{r}$ & $p$-value \\
\hline \multicolumn{5}{|l|}{ Demographic } \\
\hline Age & -0.17 & 0.0001 & -0.13 & 0.0005 \\
\hline Education & -0.15 & 0.0001 & -0.08 & 0.0365 \\
\hline \multicolumn{5}{|l|}{ Covid-19 measures } \\
\hline Length of Isolation & 0.03 & 0.4149 & 0.01 & 0.7625 \\
\hline Perceive impact of the quarantine & 0.19 & 0.0001 & 0.15 & 0.0001 \\
\hline \multicolumn{5}{|l|}{ Other measures } \\
\hline Sleep quality & 0.25 & 0.0001 & 0.28 & 0.0001 \\
\hline Loneliness & 0.48 & 0.0000 & 0.27 & 0.0000 \\
\hline Behavioural Activation & -0.29 & 0.0000 & -0.16 & 0.0000 \\
\hline Experiential Avoidance & 0.35 & 0.0000 & 0.20 & 0.0000 \\
\hline Reappraisal & -0.04 & 0.2511 & -0.04 & 0.2314 \\
\hline Catastrophisation & 0.27 & 0.0001 & 0.18 & 0.0001 \\
\hline Worry & 0.45 & 0.0001 & 0.38 & 0.0001 \\
\hline Jumping to conclusion bias & 0.37 & 0.0001 & 0.21 & 0.0001 \\
\hline Anxiety & 0.45 & 0.0001 & 0.44 & 0.0001 \\
\hline Depression & 048 & 0.0001 & 0.33 & 0.0001 \\
\hline Stress & 0.40 & 0.0001 & 0.35 & 0.0001 \\
\hline
\end{tabular}


Hallucination

Note: Benjamini and Hochberg (1995) corrected significance level $p<0.04$ 


\section{Table 4.}

Group comparisons for Paranoia and Hallucinations

Paranoia

Hallucination

Demographic

Sex

$\mathrm{W}=46906, \mathrm{p}$-value $=0.673 \quad \mathrm{~W}=51850, \mathrm{p}$-value $=$

Clinical

Diagnosis of Serious Mental Disorder

$\mathrm{W}=15064$, $\mathrm{p}$-value $<$ 0.0001

$\mathrm{W}=39311, \mathrm{p}$-value $=0.003 \mathrm{~W}=37995, \mathrm{p}$-value $=$

$\mathrm{W}=29844, \mathrm{p}$-value $<$

0.0001

$\mathrm{W}=21939, \mathrm{p}$-value $<$

0.0001

$\mathrm{W}=9415, \mathrm{p}$-value $=0.349$
$\mathrm{W}=15762, \mathrm{p}$-value $<$ 0.0001

$\mathrm{W}=29843, \mathrm{p}$-value $=$ 0.0006

$\mathrm{W}=25889, \mathrm{p}-$ value $=$

$\mathrm{W}=8841, \mathrm{p}$-value $=0$

Covid-19 measures

Familiy diagnosed COVID-19

$\mathrm{W}=47028, \mathrm{p}$-value $=0.742 \quad \mathrm{~W}=41230, \mathrm{p}$-value $=$

COVID-19 symptoms

$\mathrm{W}=27166, \mathrm{p}$-value $=0.810 \quad \mathrm{~W}=23817, \mathrm{p}$-value $=$

Note: Benjamini and Hochberg (1995) corrected significance level $p<0.03$ 


\section{Table 5.}

Regression Models for Paranoia.

\section{Paranoia}

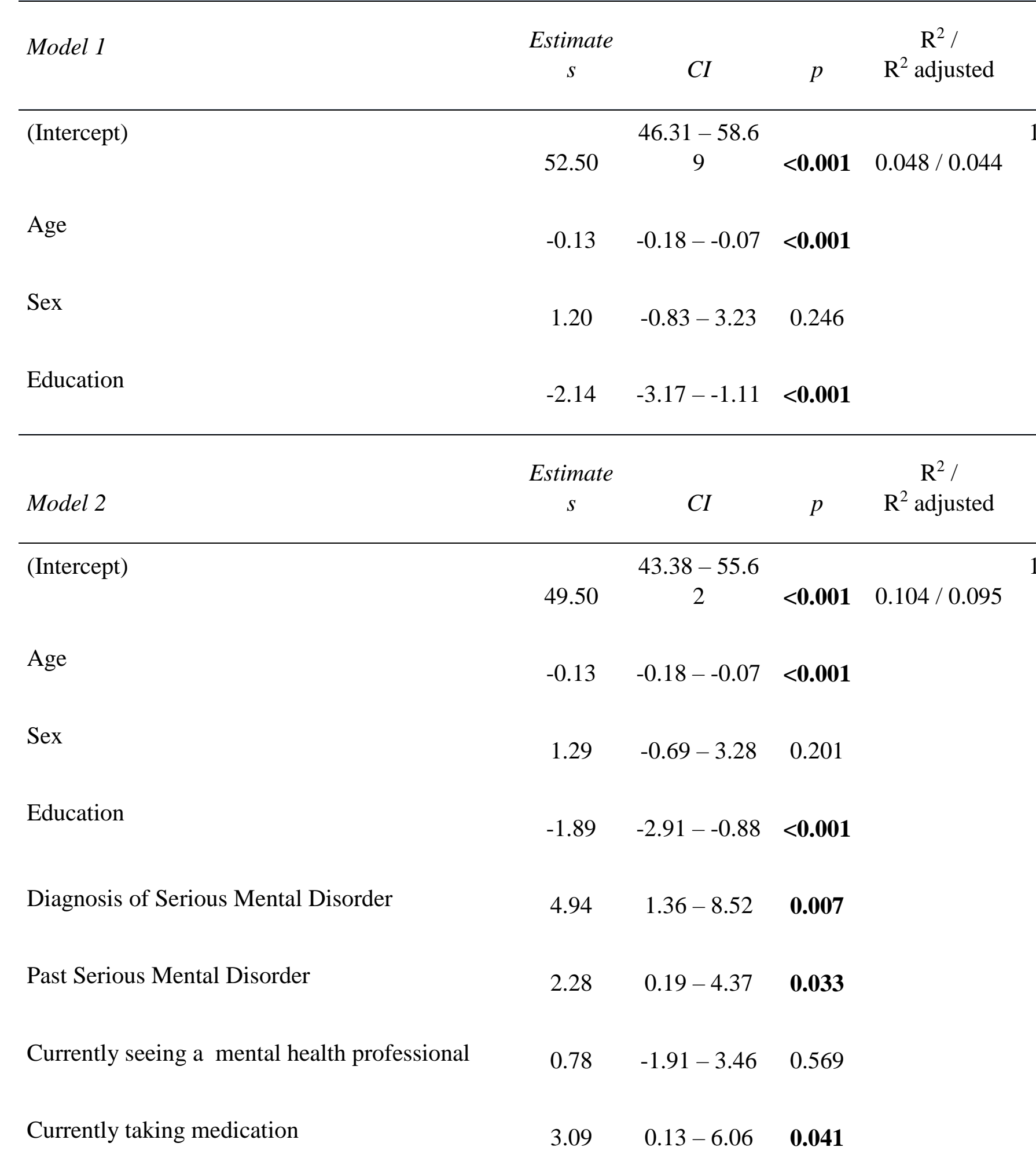




\begin{tabular}{|c|c|c|c|c|}
\hline Model3 - Covid-19 triggers & $\begin{array}{c}\text { Estimate } \\
s\end{array}$ & $C I$ & $p$ & $\begin{array}{c}\mathrm{R}^{2} / \\
\mathrm{R}^{2} \text { adjusted }\end{array}$ \\
\hline (Intercept) & 39.31 & $\begin{array}{c}32.17-46.4 \\
4\end{array}$ & $<0.001$ & $0.137 / 0.127$ \\
\hline Age & -0.12 & $-0.17--0.07$ & $<0.001$ & \\
\hline Sex & 1.44 & $-0.51-3.39$ & 0.148 & \\
\hline Education & -1.83 & $-2.83--0.83$ & $<0.001$ & \\
\hline Diagnosis of Serious Mental Disorder & 4.66 & $1.15-8.18$ & 0.009 & \\
\hline Past Serious Mental Disorder & 2.32 & $0.26-4.37$ & 0.027 & \\
\hline Currently seeing a mental health professional & 0.67 & $-1.97-3.30$ & 0.621 & \\
\hline Currently taking medication & 2.62 & $-0.30-5.53$ & 0.078 & \\
\hline Perceived impact of the quarantine & 0.42 & $0.26-0.57$ & $<0.001$ & \\
\hline Model 4-Contextual triggers & $\begin{array}{c}\text { Estimate } \\
s\end{array}$ & $C I$ & $p$ & $\begin{array}{c}\mathrm{R}^{2} / \\
\mathrm{R}^{2} \text { adjusted }\end{array}$ \\
\hline (Intercept) & 24.32 & $\begin{array}{c}14.98-33.6 \\
5\end{array}$ & $<0.001$ & $0.317 / 0.306$ \\
\hline Age & -0.14 & $-0.19--0.09$ & $<0.001$ & \\
\hline Sex & 1.29 & $-0.46-3.03$ & 0.148 & \\
\hline Education & -1.47 & $-2.36--0.57$ & 0.001 & \\
\hline Diagnosis of Serious Mental Disorder & 1.23 & $-1.95-4.41$ & 0.447 & \\
\hline
\end{tabular}


Past Serious Mental Disorder

Currently seeing a mental health professional

Currently taking medication

Perceived impact of the quarantine

Sleep quality

Behavioural Activation

Loneliness

$0.04-0.12-0.20$

0.642

$0.61 \quad 0.09-1.13$

0.021

$-0.06$

$-0.16-0.04$

0.231

0.58

$0.48-0.68$

$<0.001$

Model 5 - Cognitive Factors

\section{Estimate}

$S$

11.60

$1.83-21.37$

$\mathbf{0 . 0 2 0}$

$0.358 / 0.347$

Age

Sex

Education

Diagnosis of Serious Mental Disorder

Past Serious Mental Disorder

Currently seeing a mental health professional

Currently taking medication

Perceived impact of the quarantine
$-0.11-0.15--0.06$

$<0.001$

2.51

$0.79-4.24$

0.004

$-1.64$

$-2.51--0.77$

$<0.001$

1.22

$-1.86-4.31$

0.437

1.98

$0.19-3.76$

0.030

$-0.02$

$-2.31-2.26$

0.985

0.89

$-1.72-3.50$

0.503

0.01

$-0.14-0.17$

0.868
$\mathrm{R}^{2} /$

$\mathrm{R}^{2}$ adjusted 


\begin{tabular}{|c|c|c|c|c|}
\hline Sleep quality & 0.60 & $0.10-1.10$ & 0.019 & \\
\hline Behavioural Activation & -0.05 & $-0.15-0.05$ & 0.297 & \\
\hline Loneliness & 0.50 & $0.40-0.60$ & $<0.001$ & \\
\hline Cognitive Bias & 1.64 & $1.16-2.11$ & $<0.001$ & \\
\hline Model 6 - Emotional factors & $\begin{array}{c}\text { Estimate } \\
s\end{array}$ & $C I$ & $p$ & $\begin{array}{c}\mathrm{R}^{2} / \\
\mathrm{R}^{2} \text { adjusted }\end{array}$ \\
\hline (Intercept) & 4.90 & $-4.81-14.62$ & 0.322 & $0.422 / 0.409$ \\
\hline Age & -0.08 & $-0.13--0.04$ & 0.001 & \\
\hline Sex & 2.81 & $1.16-4.46$ & 0.001 & \\
\hline Education & -1.21 & $-2.06--0.37$ & 0.005 & \\
\hline Diagnosis of Serious Mental Disorder & 0.19 & $-2.75-3.14$ & 0.898 & \\
\hline Past Serious Mental Disorder & 1.76 & $0.06-3.46$ & 0.042 & \\
\hline Currently seeing a mental health professional & -0.94 & $-3.15-1.27$ & 0.403 & \\
\hline Currently taking medication & 0.66 & $-1.84-3.15$ & 0.605 & \\
\hline Perceived impact of the quarantine & -0.07 & $-0.22-0.08$ & 0.372 & \\
\hline Sleep quality & 0.05 & $-0.45-0.55$ & 0.842 & \\
\hline Behavioural Activation & 0.07 & $-0.02-0.17$ & 0.140 & \\
\hline Loneliness & 0.42 & $0.32-0.51$ & $<0.001$ & \\
\hline
\end{tabular}


Jumping to conclusion Bias

Anxiety

Experiential Avoidance

Repetitive Thoughts

Catastrophisation

\section{Estimate}

$s$

9.51

$0.27-18.75$

0.044

$0.448 / 0.435$
Age

Sex

Education

Diagnosis of Serious Mental Disorder

Past Serious Mental Disorder

Currently seeing a mental health professional

Currently taking medication

Perceived impact of the quarantine

Sleep quality

Behavioral Activation
$-0.09$

$-0.13--0.04$

$<0.001$

2.92

$1.30-4.54$

$<0.001$

$-1.29$

$-2.11--0.47$

0.002

$-0.72 \quad-3.64-2.20 \quad 0.628$

$1.53-0.15-3.20 \quad 0.074$

$\begin{array}{lll}-0.89 & -3.08-1.30 & 0.424\end{array}$

$1.79 \quad-0.68-4.27 \quad 0.154$

$\begin{array}{lll}-0.07 & -0.22-0.08 & 0.385\end{array}$

$\begin{array}{lll}-0.15 & -0.64-0.35 & 0.559\end{array}$

$0.02 \quad-0.07-0.12 \quad 0.636$ 
Loneliness

Jumping to conclusion Bias

Anxiety

Experiential Avoidance

Repetitive Thoughts

Hallucinations
$0.42 \quad 0.32-0.51 \quad<\mathbf{0 . 0 0 1}$

$1.28 \quad 0.83-1.74 \quad<0.001$

$0.59 \quad 0.31-0.87$

$<0.001$

$0.09 \quad 0.01-0.16$

$\mathbf{0 . 0 2 3}$

$0.25 \quad-0.12-0.62 \quad 0.190$

$0.54 \quad 0.36-0.72<\mathbf{0 . 0 0 1}$

Note: $* * * p<0.0001$ 
Table 6.

Regression Models for Hallucinations.

\section{Hallucinations}

\begin{tabular}{|c|c|c|c|c|c|}
\hline Model 1 & Estimates & $C I$ & $p$ & $\mathrm{R}^{2} / \mathrm{R}^{2}$ adjusted & $\mathrm{F}$ \\
\hline (Intercept) & 7.77 & $5.56-9.99$ & $<0.001$ & $0.027 / 0.023$ & $6.58 * * *$ \\
\hline Age & -0.03 & $-0.05--0.02$ & 0.001 & & \\
\hline Sex & -0.59 & $-1.32-0.13$ & 0.108 & & \\
\hline Education & -0.41 & $-0.77--0.04$ & 0.031 & & \\
\hline Model 2 & Estimates & $C I$ & $p$ & $\mathrm{R}^{2} / \mathrm{R}^{2}$ adjusted & $\mathrm{F}$ \\
\hline (Intercept) & 6.91 & $4.70-9.12$ & $<0.001$ & $0.068 / 0.058$ & $7.31 * * *$ \\
\hline Age & -0.03 & $-0.05--0.01$ & 0.002 & & \\
\hline Sex & -0.57 & $-1.28-0.15$ & 0.122 & & \\
\hline Education & -0.36 & $-0.72-0.01$ & 0.056 & & \\
\hline $\begin{array}{l}\text { Diagnosis of Serious Mental } \\
\text { Disorder }\end{array}$ & 2.38 & $1.08-3.67$ & $<0.001$ & & \\
\hline Past Serious Mental Disorder & 0.80 & $0.05-1.56$ & 0.038 & & \\
\hline $\begin{array}{l}\text { Currently seeing a mental } \\
\text { health professional }\end{array}$ & 0.14 & $-0.84-1.12$ & 0.777 & & \\
\hline Currently taking medication & -0.33 & $-1.41-0.75$ & 0.544 & & \\
\hline Model3 - Covid-19 triggers & Estimates & $C I$ & $p$ & $\mathrm{R}^{2} / \mathrm{R}^{2}$ adjusted & $\mathrm{F}$ \\
\hline (Intercept) & 4.33 & $1.73-6.93$ & 0.001 & $0.085 / 0.074$ & $8.14 * * *$ \\
\hline Age & -0.03 & $-0.05--0.01$ & 0.003 & & \\
\hline
\end{tabular}


Sex

Education

Diagnosis of Serious Mental

Disorder

Past Serious Mental Disorder

Currently seeing a mental

health professional

Currently taking medication

Perceived impact of the quarantine
$-0.53 \quad-1.24-0.18$

0.142

$-0.34$

$-0.70-0.02$

0.067

2.30

$1.01-3.59$

$<0.001$

0.81

$0.06-1.56$

0.034

0.12

$-0.86-1.09$

0.816

$-0.45$

$-1.52-0.63$

0.415
0.11
$0.05-0.16$
$<0.001$

\begin{tabular}{lccccc} 
Model 4-Contextual triggers & Estimates & $C I$ & $p$ & $\mathrm{R}^{2} / \mathrm{R}^{2}$ adjusted & $\mathrm{F}$ \\
\hline (Intercept) & 0.53 & $-3.13-4.19$ & 0.775 & $0.158 / 0.145$ & $12.00^{* * * * *}$ \\
& & & & \\
Age & -0.03 & $-0.05--0.01$ & $\mathbf{0 . 0 0 2}$ & \\
Sex & -0.50 & $-1.19-0.18$ & 0.148 &
\end{tabular}

Education

$-0.28 \quad-0.63-0.07$

0.118

Diagnosis of Serious Mental

Disorder

1.78

$0.53-3.04$

0.005

Past Serious Mental Disorder

0.69

$-0.03-1.41$

0.062

Currently seeing a mental health professional

0.04

$-0.90-0.97$

0.939

Currently taking medication

$$
-1.07
$$

$-2.13--0.01$

0.048

Perceived impact of the quarantine

0.02

$-0.04-0.09$

0.470

Sleep quality

0.53

$0.33-0.74$

$<0.001$ 


\begin{tabular}{|c|c|c|c|c|c|}
\hline Behavioural Activation & 0.02 & $-0.02-0.06$ & 0.431 & & \\
\hline Loneliness & 0.10 & $0.06-0.13$ & $<0.001$ & & \\
\hline Model 5 - Cognitive Factors & Estimates & $C I$ & $p$ & $\mathrm{R}^{2} / \mathrm{R}^{2}$ adjusted & $\mathrm{F}$ \\
\hline (Intercept) & -1.10 & $-5.05-2.84$ & 0.583 & $0.164 / 0.150$ & $11.45^{* *}$ \\
\hline Age & -0.03 & $-0.05--0.01$ & 0.008 & & \\
\hline Sex & -0.35 & $-1.04-0.35$ & 0.331 & & \\
\hline Education & -0.30 & $-0.65-0.05$ & 0.091 & & \\
\hline $\begin{array}{l}\text { Diagnosis of Serious Mental } \\
\text { Disorder }\end{array}$ & 1.78 & $0.53-3.03$ & 0.005 & & \\
\hline Past Serious Mental Disorder & 0.69 & $-0.03-1.41$ & 0.061 & & \\
\hline $\begin{array}{l}\text { Currently seeing a mental } \\
\text { health professional }\end{array}$ & -0.01 & $-0.94-0.92$ & 0.983 & & \\
\hline Currently taking medication & -1.15 & $-2.21--0.08$ & 0.034 & & \\
\hline $\begin{array}{l}\text { Perceived impact of the } \\
\text { quarantine }\end{array}$ & 0.02 & $-0.04-0.08$ & 0.527 & & \\
\hline Sleep quality & 0.53 & $0.33-0.73$ & $<0.001$ & & \\
\hline Behavioural Activation & 0.02 & $-0.02-0.06$ & 0.393 & & \\
\hline Loneliness & 0.09 & $0.05-0.13$ & $<0.001$ & & \\
\hline Cognitive bias & 0.21 & $0.02-0.40$ & $\mathbf{0 . 0 3 2}$ & & \\
\hline Model 6 - Emotional Factors & Estimates & $C I$ & $p$ & $\mathrm{R}^{2} / \mathrm{R}^{2}$ adjusted & $\mathrm{F}$ \\
\hline (Intercept) & -2.73 & $-6.64-1.19$ & 0.172 & $0.247 / 0.231$ & $14.4^{* * *}$ \\
\hline Age & -0.01 & $-0.03-0.00$ & 0.150 & & \\
\hline Sex & -0.17 & $-0.84-0.49$ & 0.608 & & \\
\hline
\end{tabular}


Education

Diagnosis of Serious Mental

Disorder

Past Serious Mental Disorder

Currently seeing a mental health professional

Currently taking medication

Perceived impact of the quarantine

Sleep quality

Behavioural Activation

Loneliness

Cognitive bias

Repetitive Thoughts

Experiential Avoidance

Anxiety

Catastrophisation
$-0.19$

1.42

$0.23-2.62$

0.61

$-0.40$

$-1.30-0.50$

0.389

$-1.24 \quad-2.25--0.23$

0.016

$-0.00 \quad-0.06-0.06$

0.956

0.31

$0.11-0.51$

0.003

0.06

$0.02-0.10$

0.002

0.06

$0.02-0.10$

0.003

0.06

$-0.13-0.24$

0.558

0.05

$0.02-0.08$

0.003

0.01

$-0.01-0.04$

0.260

0.37

$0.26-0.48$

$<0.001$

$-0.06$

$-0.21-0.09$

0.456

\begin{tabular}{lccccc}
\hline Model 7 & Estimates & $C I$ & $p$ & $\mathrm{R}^{2} / \mathrm{R}^{2}$ adjusted & $\mathrm{F}$ \\
\hline (Intercept) & -3.17 & $-7.00-0.66$ & 0.104 & $0.283 / 0.266$ & $16.17^{* *}$ \\
Age & -0.01 & $-0.02-0.01$ & 0.551 & \\
Sex & -0.41 & $-1.07-0.25$ & 0.219 & \\
Education & -0.08 & $-0.41-0.26$ & 0.644
\end{tabular}


Diagnosis of Serious Mental

Disorder

Past Serious Mental Disorder

Currently seeing a mental

health professional

Currently taking medication

Perceived impact of the quarantine

Sleep quality

Behavioural Activation

Loneliness

Cognitive bias

Repetitive Thoughts

Experiential Avoidance

Anxiety

Catastrophisation

Paranoia

$-0.02-0.06$

0.264

$\begin{array}{lll}-0.05 & -0.24-0.13 & 0.573\end{array}$

$\begin{array}{lll}0.04 & 0.01-0.07 & \mathbf{0 . 0 1 3}\end{array}$

$\begin{array}{lll}0.01 & -0.02-0.03 & 0.564\end{array}$

0.30

$0.19-0.42$

$<0.001$

Note: $* * * p<0.0001$

$\begin{array}{lll}-0.08 & -0.22-0.07 & 0.315\end{array}$

0.09

$0.06-0.12$

$<0.001$ 